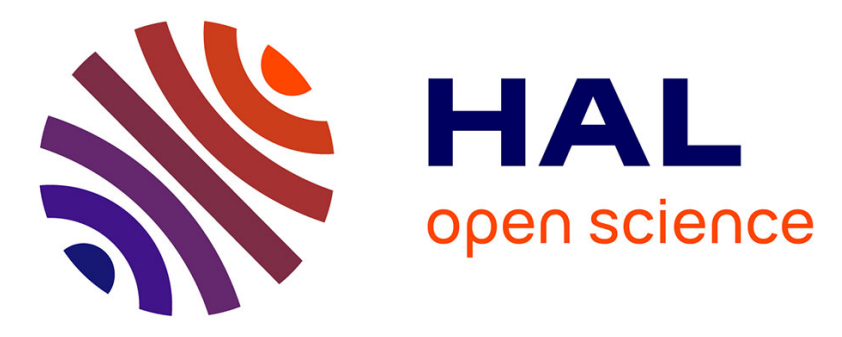

\title{
Vibrations of post-buckled rods: the singular inextensible limit
}

Sebastien Neukirch, Joel Frelat, Alain Goriely, Corrado Maurini

\section{To cite this version:}

Sebastien Neukirch, Joel Frelat, Alain Goriely, Corrado Maurini. Vibrations of post-buckled rods: the singular inextensible limit. Journal of Sound and Vibration, 2012, 331, pp.704-720. 10.1016/j.jsv.2011.09.021 . hal-00667565

\section{HAL Id: hal-00667565 https://hal.sorbonne-universite.fr/hal-00667565}

Submitted on 8 Feb 2012

HAL is a multi-disciplinary open access archive for the deposit and dissemination of scientific research documents, whether they are published or not. The documents may come from teaching and research institutions in France or abroad, or from public or private research centers.
L'archive ouverte pluridisciplinaire HAL, est destinée au dépôt et à la diffusion de documents scientifiques de niveau recherche, publiés ou non, émanant des établissements d'enseignement et de recherche français ou étrangers, des laboratoires publics ou privés. 


\title{
Vibrations of post-buckled rods: the singular inextensible limit
}

\author{
Sébastien Neukirch ${ }^{\mathrm{a}, \mathrm{b}}$, Joël Frelat ${ }^{\mathrm{a}, \mathrm{b}}$, Alain Goriely ${ }^{\mathrm{c}}$, Corrado Maurini ${ }^{\mathrm{a}, \mathrm{b}}$ \\ ${ }^{a}$ CNRS, UMR 7190, Institut Jean Le Rond d'Alembert, F-75005 Paris, France. \\ ${ }^{b}$ UPMC Univ Paris 06, UMR 7190, Institut Jean Le Rond d'Alembert, F-75005 Paris, France. \\ ${ }^{c}$ Oxford Centre for Collaborative Applied Mathematics (OCCAM), Oxford University, U.K.
}

\begin{abstract}
The small-amplitude in-plane vibrations of an elastic rod clamped at both extremities are studied. The rod is modeled as an extensible, shearable, planar Kirchhoff elastic rod under large displacements and rotations, and the vibration frequencies are computed both analytically and numerically as a function of the loading. Of particular interest is the variation of mode frequencies as the load is increased through the buckling threshold. While for some modes there are no qualitative changes in the mode frequencies, other frequencies experience rapid variations after the buckling threshold, the thinner the rod, the more abrupt the variations. Eventually, a mismatch for half of the frequencies at buckling arises between the zero thickness limit of the extensible model and the inextensible model.
\end{abstract}

Keywords: Vibrations, Kirchhoff elastic rods, Buckling, Bifurcation.

\section{Introduction}

The first step in the study of vibrating elastic structures [1] focuses on the dynamical response of the system around its unstressed configuration. In vibration analysis, the dynamics of infinitely small-amplitude disturbances around the fundamental equilibrium state are generally first considered, leading to a linear problem. However, nonlinear effects are known to play a key role in many elastic systems, and in the context of vibrations, nonlinearities can be included by studying large-amplitude oscillations around the fundamental state [2]. The second step in the analysis of vibrations is to study the effect of external loads. They have a direct influence on the dynamical response of the system, as easily demonstrated by tuning the natural frequencies of a string by putting it under tension. Similarly, in compression, the natural frequencies of a rod decrease. Again nonlinear effects become important when external loads not only change the vibration response of the rod but also alter its overall stability through buckling. Several studies have investigated dynamical responses of post-buckled elastic rods around their post-buckled state, see e.g. Chap. 12 of [1] and $[3,4,5]$. The present work also focuses on the problem of small-amplitude vibrations around a pre-strained deformed nonlinear elastic rod, and shows that vibration frequencies behave singularly at buckling. This problem is relevant for a number of applications including the manufacturing of piano (or violin) soundboards where the wooden board is bent before being clamped in the rigid metal frame $[6,7,8]$. Other systems where prestress and/or pre-strain play an important role for the vibration response are gongs, cymbals, and steel drums where plastic deformations of the metal plates are used to introduce separation of response modes [9]. 
2. Model

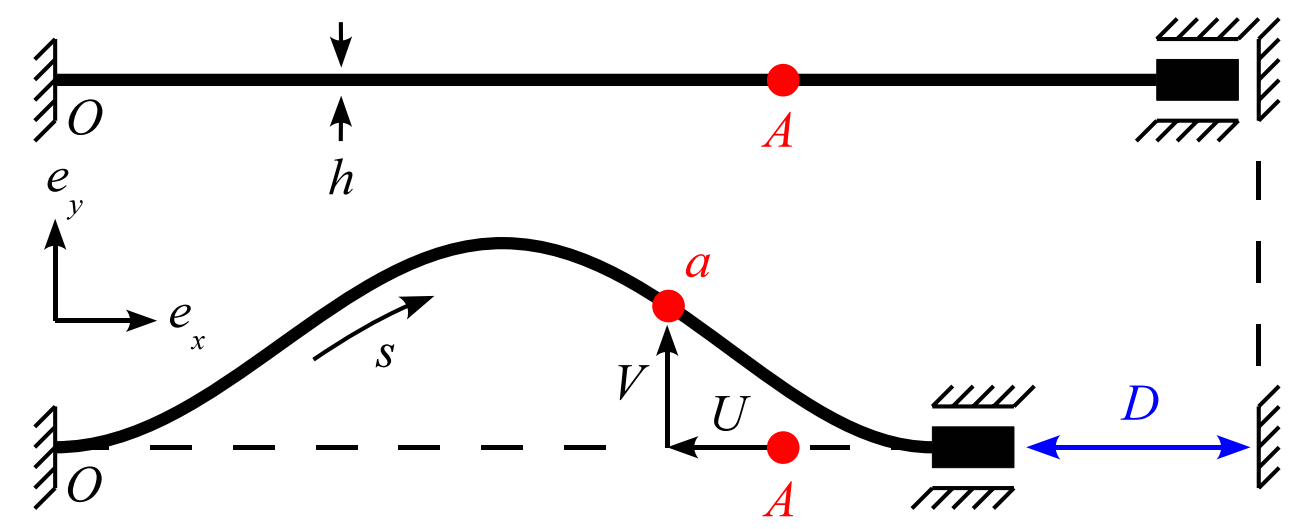

Here, we consider the problem of in-plane vibrations of a pre- and post-buckled Kirchhoff extensible shearable elastic rod. First, we study the equilibrium configurations of a clampedclamped rod as the ends are gradually moved together. The rod has a straight natural shape and for small axial displacements the rod remains straight until a critical displacement is reached where the rod buckles in the plane. For each value of the axial displacement we study the smallamplitude vibrations around the equilibrium state and we follow how frequencies of the natural modes change as the load is increased. For both equilibrium and vibrations we enforce displacement control boundary conditions, that is, the axial displacement rather than the axial load is imposed.

The paper is organized as follows: In Section 2 we present Kirchhoff model for elastic rods, in Section 3 we derive the equations for the small-amplitude vibrations of a rod around its postbuckled equilibrium. We compute equilibriums in Section 4, vibrations in the extensible case in Section 5 and in the extensible case in Section 6. We then compare the two cases in Section 7 and a show a discrepancy between them, which is further analyzed analytically in Section 8. Discussion (Section 9) and conclusion (Section 10) follow.

Figure 1: Clamped-clamped rod buckled in the $(x, y)$ plane. The end-shortening D is controlled. The point $A$ in the reference configuration moves to point $a$ in the deformed configuration, introducing horizontal $U \leq 0$ and vertical $V$ displacements. The origin is taken at the fixed point $O$ at the left end of the rod.

We consider an elastic rod with a rectangular cross-section of width $b$ and thickness $h$, total length $L$ and arc length $S$ in its unstressed reference state. In this state the rod lies along the $\boldsymbol{e}_{\boldsymbol{x}}$ axis, from the origin $O=(0,0,0)$ to the point at $(L, 0,0)$. The position vector of the center of the rod cross-section is noted $\boldsymbol{R}(S)$ and we have $\boldsymbol{R}(0)=(0,0,0)$ and $\boldsymbol{R}(L)=(L, 0,0)$ in the reference state.

\section{Kinematics}

We use the special Cosserat theory of rods [10] where the rod can suffer bending, extension, and shear deformations. We work under the assumption that the rod cross-section remains planar (and rectangular) as the rod deforms and use a set of three Cosserat directors $\left(\boldsymbol{d}_{\mathbf{1}}(S), \boldsymbol{d}_{\mathbf{2}}(S), \boldsymbol{d}_{\mathbf{3}}(S)\right)$ 
embedded in each cross-section: $\boldsymbol{d}_{\mathbf{1}}$ is perpendicular to the section plane, $\boldsymbol{d}_{\mathbf{2}}$ is along the small span (of length $h$ ) of the section, and $\boldsymbol{d}_{3}$ is along the wide span (of length $b$ ) of the section. In the 49 undeformed state, $\boldsymbol{d}_{\mathbf{1}}(S) \equiv \boldsymbol{e}_{\boldsymbol{x}}, \boldsymbol{d}_{\mathbf{2}}(S) \equiv \boldsymbol{e}_{\boldsymbol{y}}$, and $\boldsymbol{d}_{\mathbf{3}}(S) \equiv \boldsymbol{e}_{z}$. We only consider deformed states 5o that are (i) planar (where the rod center line $\boldsymbol{R}(S)$ lies in the $(x, y)$ plane, the rod being bent along ${ }_{51}$ its small span $h$ ), and (ii) twist-less (where the director $\boldsymbol{d}_{\mathbf{3}}(S) \equiv \boldsymbol{e}_{z}$ ). Note that in the presence of 52 extension and shear, $S$ may no longer be the arc-length of the curve $\boldsymbol{R}(S)$ in the deformed state.

53 We introduce extension and shear strains, $e_{1}$ and $e_{2}$, such that:

$$
\boldsymbol{R}^{\prime}(S) \stackrel{\text { def }}{=} \mathrm{d} \boldsymbol{R} / \mathrm{d} S=\left(1+e_{1}\right) \boldsymbol{d}_{\mathbf{1}}+e_{2} \boldsymbol{d}_{\mathbf{2}} .
$$

In the absence of extension $\left(e_{1}=0\right)$ and shear $\left(e_{2}=0\right)$, the director $\boldsymbol{d}_{1}$ is the unit tangent to the ${ }_{55}$ centerline $\boldsymbol{R}(S)=(X(S), Y(S), Z(S))$. We introduce the angle $\theta(S)$ to parametrize the rotation of the $\left(\boldsymbol{d}_{\mathbf{1}}, \boldsymbol{d}_{\mathbf{2}}\right)$ frame around the $\boldsymbol{e}_{z}=\boldsymbol{d}_{\mathbf{3}}$ axis:

$$
\boldsymbol{d}_{\mathbf{1}}(S)=\left(\begin{array}{c}
\cos \theta(S) \\
\sin \theta(S) \\
0
\end{array}\right)_{\boldsymbol{e}_{\boldsymbol{x}}, \boldsymbol{e}_{\boldsymbol{y}}, \boldsymbol{e}_{z}} \text { and } \quad \boldsymbol{d}_{\mathbf{2}}(S)=\left(\begin{array}{c}
-\sin \theta(S) \\
\cos \theta(S) \\
0
\end{array}\right)_{\boldsymbol{e}_{\boldsymbol{x}}, \boldsymbol{e}_{\boldsymbol{y}}, \boldsymbol{e}_{z}}
$$

57 Dynamics

We use the Kirchhoff dynamical equations for elastic rods [10], where the stresses in the 59 section are averaged to yield an internal force $N(S)$ and an internal moment $\boldsymbol{M}(S)$. These internal ${ }_{60}$ forces and moments are the loads exerted on the section at $S$ by the part of the rod at $\bar{S}>S$. In 61 the absence of body force and couple, the linear and angular momentum balance then read

$$
\begin{aligned}
N^{\prime}(S, T) & =\rho h b \ddot{\boldsymbol{R}}(S, T), \\
\boldsymbol{M}^{\prime}(S, T)+\boldsymbol{R}^{\prime}(S, T) \times N(S, T) & =\rho I \ddot{\theta}(S, T),
\end{aligned}
$$

62 where ()$^{\prime} \stackrel{\text { def }}{=} \partial / \partial S,(j) \stackrel{\text { def }}{=} \partial / \partial T, T$ is time, $\rho$ the mass per unit volume of the material, and $I$ the ${ }_{63}$ second moment of area of the cross-section (in the present case $I=h^{3} b / 12$ ).

64 Constitutive law

65 We use the standard linear constitutive relationship relating the bending strain $\kappa(S) \stackrel{\text { def }}{=} \theta^{\prime}(S)$ 66 to the bending moment $M_{3} \stackrel{\text { def }}{=} \boldsymbol{M} \cdot \boldsymbol{d}_{\mathbf{3}}$ :

$$
M_{3}=E I \kappa,
$$

${ }_{67}$ where $E$ is Young's modulus. Note that $\kappa$ is not the curvature in general. In a similar way, the 68 tension $N_{1} \stackrel{\text { def }}{=} \boldsymbol{N} \cdot \boldsymbol{d}_{\mathbf{1}}$ and the shear force $N_{2} \stackrel{\text { def }}{=} \boldsymbol{N} \cdot \boldsymbol{d}_{\mathbf{2}}$ are linked to the extension $e_{1}$ and shear 69 strains $e_{2}$ through

$$
\begin{aligned}
& N_{1}=E h b e_{1}, \\
& N_{2}=G h b e_{2} .
\end{aligned}
$$

70 where $G$ is the shear modulus. 
Equations in component form

In the planar case considered here, we have $Z(S, T) \equiv 0, N_{z}(S, T) \equiv 0, M_{x}(S, T) \equiv 0$, and $M_{y}(S, T) \equiv 0, \forall(S, T)$ so that the equations for the six remaining unknowns are

$$
\begin{aligned}
X^{\prime} & =\left(1+e_{1}\right) \cos \theta-e_{2} \sin \theta, \\
Y^{\prime} & =\left(1+e_{1}\right) \sin \theta+e_{2} \cos \theta, \\
\theta^{\prime} & =M /(E I), \\
M^{\prime} & =e_{2} N_{1}-\left(1+e_{1}\right) N_{2}+\rho I \ddot{\theta}, \\
N_{x}^{\prime} & =\rho h b \ddot{X}, \\
N_{y}^{\prime} & =\rho h b \ddot{Y},
\end{aligned}
$$

where $M=M_{z}=M_{3}, N_{1}=N_{x} \cos \theta+N_{y} \sin \theta$, and $N_{2}=-N_{x} \sin \theta+N_{y} \cos \theta$. The strains $\left(e_{1}, e_{2}\right)$ are given by Eqs (6) and (7) as functions of $N_{x}$ and $N_{y}$.

\section{Dimensionless variables}

We scale all lengths with $L$, time with $\tau \stackrel{\text { def }}{=} L^{2} \sqrt{\rho h b /(E I)}$, forces with $E I / L^{2}$, and moments with $E I / L$. This naturally introduces a parameter

$$
\eta \stackrel{\text { def }}{=} \frac{I}{h b L^{2}}=\frac{1}{12}\left(\frac{h}{L}\right)^{2},
$$

79

which takes small values in the present case of slender rods. Dimensionless variables will be written lowercase, e.g. $x \stackrel{\text { def }}{=} X / L$, or $m \stackrel{\text { def }}{=} M L /(E I)$. The constitutive relations (6) and (7) read: $e_{1}=\eta n_{1}$ and $e_{2}=2(1+v) \eta n_{2}$, where the Poisson ratio $v$ arises from the relation $E=2(1+v) G$.

Rods with $\eta>0$ are extensible, shearable rods for which the rotational inertia is accounted for. They will be simply called extensible rods. Rods with $\eta=0$ will be simply called inextensible rods, although they really are inextensible, unshearable rods for which the rotational inertia is ignored. Such rods are frequently called elastica $[16,17]$.

\section{Small-amplitude vibrations around pre- and post-buckled equilibrium}

The systems of equations (8) in dimensionless form reads

$$
\begin{aligned}
x^{\prime}(s, t) & =\cos \theta+\eta\left(n_{1} \cos \theta-2(1+v) n_{2} \sin \theta\right), \\
y^{\prime}(s, t) & =\sin \theta+\eta\left(n_{1} \sin \theta+2(1+v) n_{2} \cos \theta\right), \\
\theta^{\prime}(s, t) & =m, \\
m^{\prime}(s, t) & =-n_{2}+\eta\left((1+2 v) n_{1} n_{2}+\ddot{\theta}\right), \\
n_{x}^{\prime}(s, t) & =\ddot{x}, \\
n_{y}^{\prime}(s, t) & =\ddot{y},
\end{aligned}
$$

with $n_{1}=n_{x} \cos \theta+n_{y} \sin \theta$ and $n_{2}=-n_{x} \sin \theta+n_{y} \cos \theta$. In our problem, we consider a clampedclamped rod and control the end-shortening $d \stackrel{\text { def }}{=} 1-(x(1, t)-x(0, t))$. This setup implies the following boundary conditions:

$$
\begin{array}{ll}
x(0, t)=0 & x(1, t)=1-d, \\
y(0, t)=0 & y(1, t)=0, \\
\theta(0, t)=0 & \theta(1, t)=0 . \\
& 4
\end{array}
$$


For each given value of the end-shortening $d$, we find the equilibrium configuration $\left(x_{e}, y_{e}, \theta_{e}\right.$, $m_{e}, n_{x e}, n_{y e}$ ) by solving system (10) with $\ddot{x}_{e}=0$ and $\ddot{y}_{e}=0$. Then we look for small-amplitude vibrations around this equilibrium configuration, that is, we set

$$
\begin{aligned}
x(s, t) & =x_{e}(s)+\delta \bar{x}(s) \mathrm{e}^{\mathrm{i} \omega t}, \\
y(s, t) & =y_{e}(s)+\delta \bar{y}(s) \mathrm{e}^{\mathrm{i} \omega t}, \\
\theta(s, t) & =\theta_{e}(s)+\delta \bar{\theta}(s) \mathrm{e}^{\mathrm{i} \omega t}, \\
m(s, t) & =m_{e}(s)+\delta \bar{m}(s) \mathrm{e}^{\mathrm{i} \omega t}, \\
n_{x}(s, t) & =n_{x e}(s)+\delta \bar{n}_{x}(s) \mathrm{e}^{\mathrm{i} \omega t}, \\
n_{y}(s, t) & =n_{y e}(s)+\delta \bar{n}_{y}(s) \mathrm{e}^{\mathrm{i} \omega t},
\end{aligned}
$$

where $\delta \ll 1$ is a small parameter, and $\omega$ is the frequency of the vibration. Inserting (12) into (10) and keeping only linear terms in $\delta$, we obtain equations for the spatial modes $\left(\bar{x}, \bar{y}, \bar{\theta}, \bar{m}, \bar{n}_{x}, \bar{n}_{y}\right)$ :

$$
\begin{aligned}
\bar{x}^{\prime}(s)= & -\bar{\theta} \sin \theta_{e}+\eta\left(\bar{n}_{1} \cos \theta_{e}-2(1+v) \bar{n}_{2} \sin \theta_{e}\right)+ \\
& \eta \bar{\theta}\left(-n_{1 e} \sin \theta_{e}-2(1+v) n_{2 e} \cos \theta_{e}\right) \\
\bar{y}^{\prime}(s)= & \bar{\theta} \cos \theta_{e}+\eta\left(\bar{n}_{1} \sin \theta_{e}+2(1+v) \bar{n}_{2} \cos \theta_{e}\right)+ \\
& \eta \bar{\theta}\left(n_{1 e} \cos \theta_{e}-2(1+v) n_{2 e} \sin \theta_{e}\right) \\
\bar{\theta}^{\prime}(s)= & \bar{m} \\
\bar{m}^{\prime}(s)= & -\bar{n}_{2}+\eta\left((1+2 v)\left(\bar{n}_{1} n_{2 e}+n_{1 e} \bar{n}_{2}\right)-\omega^{2} \bar{\theta}\right), \\
\bar{n}_{x}^{\prime}(s)= & -\omega^{2} \bar{x} \\
\bar{n}_{y}^{\prime}(s)= & -\omega^{2} \bar{y}
\end{aligned}
$$

with $\bar{n}_{1}=\bar{n}_{x} \cos \theta_{e}+\bar{n}_{y} \sin \theta_{e}+\bar{\theta}\left(-n_{x e} \sin \theta_{e}+n_{y e} \cos \theta_{e}\right)$ and $\bar{n}_{2}=-\bar{n}_{x} \sin \theta_{e}+\bar{n}_{y} \cos \theta_{e}+$ $\bar{\theta}\left(-n_{x e} \cos \theta_{e}-n_{y e} \sin \theta_{e}\right)$. The boundary conditions on the spatial modes are

$$
\begin{array}{ll}
\bar{x}(0)=0 & \bar{x}(1)=0, \\
\bar{y}(0)=0 & \bar{y}(1)=0, \\
\bar{\theta}(0)=0 & \bar{\theta}(1)=0 .
\end{array}
$$

For given parameters $\eta$ and $v$ and given end-shortening $d$, the equilibrium $\left(x_{e}, y_{e}, \theta_{e}, m_{e}, n_{x e}, n_{y e}\right)$ is first computed from (10) with $\ddot{x}_{e}=0$ and $\ddot{y}_{e}=0$. Then the 6D system (13) with the six boundary conditions (14) is a well-defined boundary value problem, but with the additional unknown $\omega$. For computational purpose, we normalize the linear solution of this problem by imposing the condition

$$
\bar{m}^{2}(0)+\bar{n}_{x}^{2}(0)+\bar{n}_{y}^{2}(0)=1 .
$$

\section{Equilibrium}

We use a 'home-made' predictor-corrector path-following code to address the problem numerically. For each value of $p \stackrel{\text { def }}{=}-n_{x e}=-N_{x e} L^{2} /(E I)$ in the interval $\left(0 ; 8 \pi^{2}\right)$, we compute the equilibrium solution $\left(x_{e}, y_{e}, \theta_{e}, m_{e}, n_{x e}, n_{y e}\right)$ satisfying clamped-clamped boundary conditions (11). 


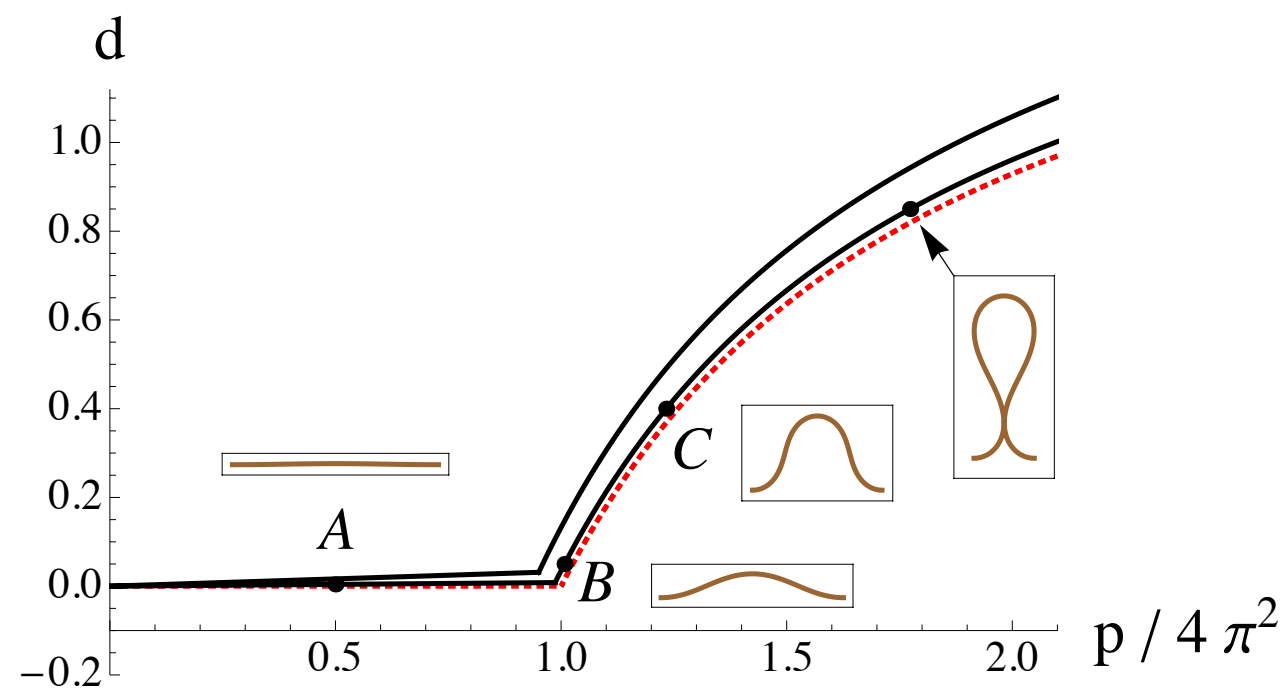

Figure 2: Fundamental and post-buckled equilibrium path of a clamped-clamped rod with $\eta=1 / 1200, \eta=1 / 4800$, and $\eta=0$ (top to bottom). As the controlled end-shortening $d=D / L$ is gradually raised, an increasing axial load $p=-N_{x e} L^{2} /(E I)$ is recorded.

In Fig. 2 equilibrium paths are given for both inextensible (i.e. $\eta=0)$ and extensible $(\eta>0)$ rods. In the latter case, we see that the displacement $d$ starts to increase as soon the curve leaves the origin: due to extensibility the rod shorten before buckling. Buckling happens at $p=4 \pi^{2}$ for the inextensible case and at lower values for extensible cases. These equilibrium paths show that extension and shear play a minor role in the buckling load and that the inextensible rod solution is obtained in the limit $\eta \rightarrow 0$ of the extensible rod solution, as expected. Closed-form formula for the dotted (red) curve is given in Appendix A.

\section{Vibrations in the extensible case}

Vibrations around the straight state

We first consider the equilibrium solution where the axially loaded rod is straight, but compressed:

$$
y_{e}(s)=0, x_{e}(s)=(1-\eta p) s, n_{y e}(s)=0, n_{x e}(s)=-p, \theta_{e}(s)=0, m_{e}(s)=0 .
$$

The vibrations around this straight equilibrium are either extensional of flexural. Extensional vibrations modes are solutions of:

$$
\begin{aligned}
& \bar{x}^{\prime}(s)=\eta \bar{n}_{x} \\
& \bar{n}_{x}^{\prime}(s)=-\omega^{2} \bar{x}
\end{aligned}
$$

with boundary conditions $\bar{x}(0)=0=\bar{x}(1)$. This yields vibrations frequencies of the form $\omega=j \pi / \sqrt{\eta}$ (with $j=1,2, \ldots$ ), which do not vary with the load $p$. The upper curve, before buckling of Fig. 3-(a) is such a solution. Flexural vibrations modes are solutions of:

$$
\bar{y}^{\prime \prime \prime \prime}+\mu_{p} \bar{y}_{6}^{\prime \prime}-\mu_{\omega}^{2} \bar{y}=0,
$$


with boundary conditions $\bar{y}(0)=0=\bar{y}(1)$ and $\left(\eta_{\star}^{2} \omega^{2}+\left[1-p\left(\eta-\eta_{\star}\right)\right]^{2}\right) \bar{y}^{\prime}(0,1)+\eta_{\star} \bar{y}^{\prime \prime \prime}(0,1)=$ 0 . We introduced the notations $\eta_{\star}=2 \eta(1+v), \mu_{p}=\left(1-p\left(\eta-\eta_{\star}\right)\right) p+\omega^{2}\left(\eta+\eta_{\star}\right)$, and $\mu_{\omega}^{2}=\omega^{2}\left(\left[1-p\left(\eta-\eta_{\star}\right)\right]^{2}-\left[1-p\left(\eta-\eta_{\star}\right)\right] p \eta_{\star}-\eta \eta_{\star} \omega^{2}\right)$. The general solution is of the form:

$$
\bar{y}(s)=A \cos k^{+} s+B \cosh k^{-} s+C \sin k^{+} s+D \sinh k^{-} s
$$

${ }_{127}$ where we used the two wave numbers $k^{ \pm}=(1 / \sqrt{2}) \sqrt{\sqrt{\mu_{p}^{2}+4 \mu_{\omega}^{2}} \pm \mu_{p}}$. The boundary conditions require that

$$
2 a^{+} a^{-} k^{+} k^{-}\left(\cos k^{+} \cosh k^{-}-1\right)+\left(\left(a^{+} k^{+}\right)^{2}-\left(a^{-} k^{-}\right)^{2}\right) \sin k^{+} \sinh k^{-}=0,
$$

with $a \pm=\left[1-p\left(\eta-\eta_{\star}\right)\right]^{2}+\eta_{\star}\left(\mp\left(k^{ \pm}\right)^{2}+\eta_{\star} \omega^{2}\right)$, which is an equation for $\omega$. The analytical solutions $\omega(p)$ given by (20) match numerical solutions given in Fig. 3-(a,b,c). Buckling occurs when $\omega=0=\mu_{\omega}$, i.e. for $k^{+}=\sqrt{\mu_{p}}=2 \pi$ and $k^{-}=0$. This yields:

$$
p=\frac{\sqrt{1+16 \pi^{2}\left(\eta_{\star}-\eta\right)}-1}{2\left(\eta_{\star}-\eta\right)}=4 \pi^{2}-16 \pi^{4}\left(\eta_{\star}-\eta\right)+O\left(\eta^{2}\right)
$$

\section{Vibrations around the buckled state}

Once the equilibrium solution is known, we solve the boundary value problem (13)-(14) numerically with a shooting method: $(i)$ we first use a guess for the unknown parameters $\chi=$ $\left(\bar{m}(0), \bar{n}_{x}(0), \bar{n}_{y}(0), \omega\right)$ and we integrate the system (13) up to $s=1$; (ii) we then check if the boundary conditions (14)-(15) are satisfied. If not, we change the guess $\chi$ accordingly (using a Newton-Raphson scheme) until the boundary conditions at $s=1$ are satisfied.
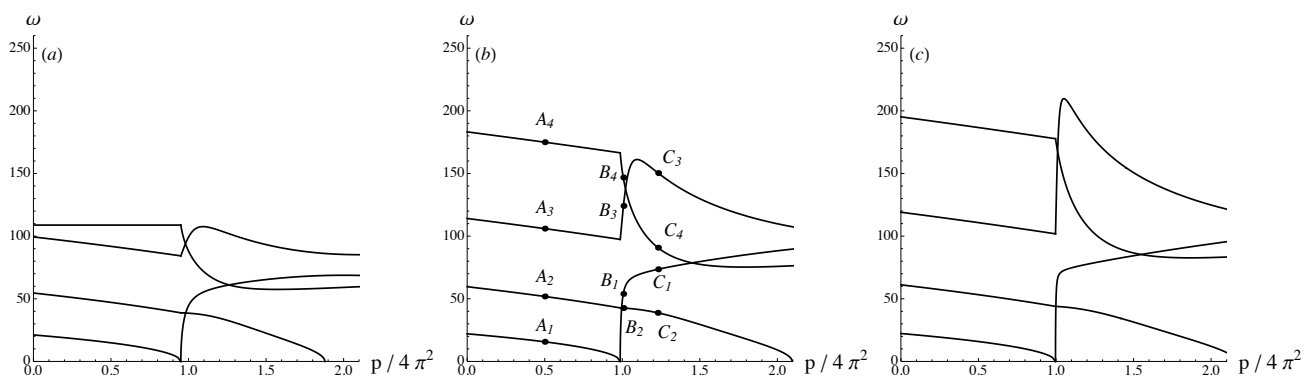

Figure 3: Frequencies for the lowest four vibration modes of a clamped-clamped rod around its fundamental and postbuckled equilibrium configurations in the extensible case: (a) for $\eta=1 / 1200$, (b) for $\eta=1 / 4800$, (c) for $\eta=1 / 19200$ (plain). The labels $A_{i}, B_{i}, C_{i}$ with $i=1,2,3,4$ correspond to the shapes given in Figs. 4, 5, 6 .

Once a solution $\chi_{i}$ is found for a given $p=p_{i}$, we set $p=p_{i+1}$ and use the value $\chi_{i}$ as starting guess (predictor step) for the shooting method at $p=p_{i+1}$ (corrector step). In this setup, each curve $\omega=\omega(p)$ represents a path in the numerical bifurcation diagram, and we have numerically computed the four first paths (i.e. lowest four curves $\omega=\omega(p)$ ) for several values of the parameter $\eta=I /\left(h w L^{2}\right)$.

In Fig. 3, frequencies for the first four modes are given as a function of the parameter $p$, for different values of $\eta$. It should be noted that the computations performed here are for a displacement control experiment, that is $d$ (and not $p$ ) is controlled. Nevertheless, as there is a one-to-one 
correspondence between $d$ and $p$, for each value of the applied longitudinal displacement $d$, the equilibrium axial load $p$ is read from Fig. 2 and then the frequency is computed and plotted in Fig. 3. We see in Fig. 3 that as $\eta \rightarrow 0$, frequencies globally increase and tend toward limiting curves. Finally we note that every curve $\omega(p)$ is continuous, but that the curves for the odd modes experience a rapid increase just after buckling. As $\eta \rightarrow 0$ this rapid increase becomes more abrupt to eventually turn into a discontinuity in the case $\eta=0$, see Section 6 . In each of Figs. 4, 5, and 6, dynamical shapes $\left(x(s, t)=x_{e}(s)+\bar{x}(s) \cos \omega t, y(s, t)=y_{e}(s)+\bar{y}(s) \cos \omega t\right)$ of the vibrating rod are plotted.
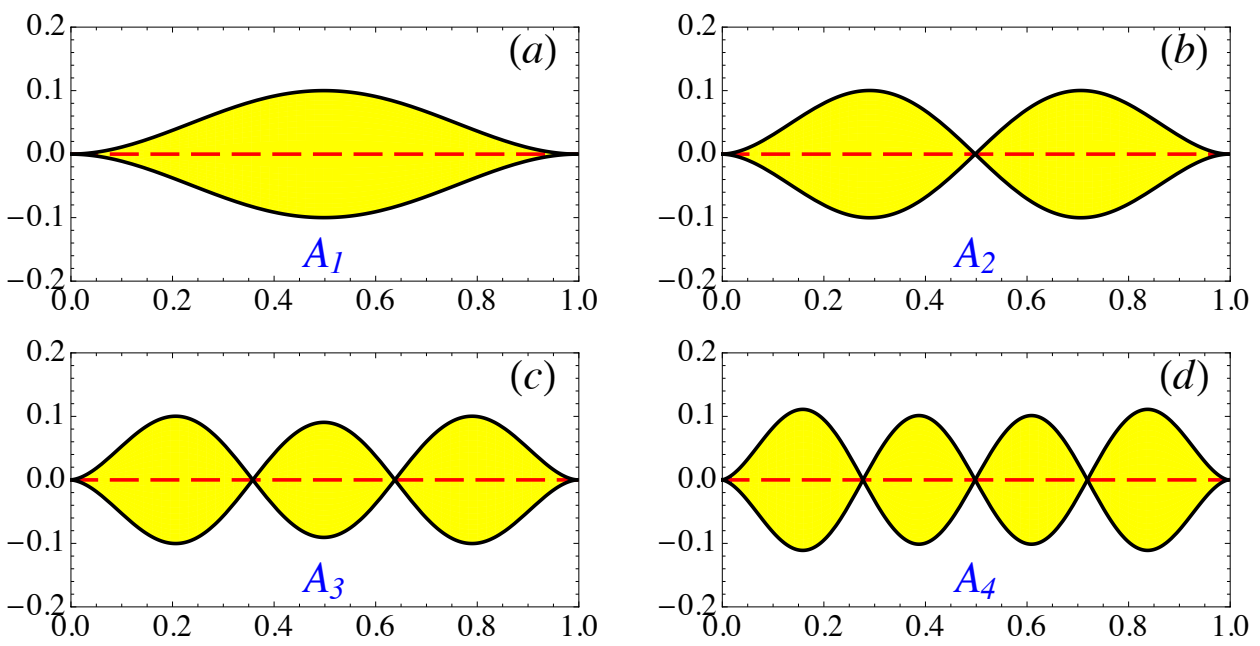

Figure 4: First four modes at $p /\left(4 \pi^{2}\right)=0.5$, for the extensible case with $\eta=1 / 4800$ : configurations $A_{1}$ (a), $A_{2}$ (b), $A_{3}$ (c), and $A_{4}$ (d) in Figure 3-(b).

We now focus on the first mode, which emerges from $\omega=0$ at buckling. In Fig. 7-(a), we plot $\omega$ as function of the rise of the buckled rod at its mid-point: $y_{e}(1 / 2)=Y_{e}(L / 2) / L$. Each curve corresponds to a different value of the parameter $\eta$, from $\eta=1 / 1200$ (i.e. $L=10 h$ ) to $\eta=1 / 480000$ (i.e. $L=200 h$ ). We see that all curves emerge from $\omega=0$ at buckling $\left(y_{e}(1 / 2)=0\right)$ and asymptotically tend to the curve computed in the inextensible case (see Section $6)$ when $y_{e}(1 / 2)$ becomes large. For very small $\eta$ values, curves rise sharply from $\omega=0$ and quickly approaches the inextensible asymptote. As a matter of fact these curves can be made to almost collapse on a master curve if the horizontal axis is plotted in unit of the rod thickness $h$ : in Fig. 7-(b) we plot the frequency $\omega$ as function of $Y_{e}(L / 2) / h$, for the same set of $\eta$ values. All curves nearly collapse on a master curve which has a (numerically determined) slope $\simeq 28$ at the origin. Using $h=\sqrt{12 \eta} L$ we obtain:

$$
\omega \simeq \frac{8.1}{\sqrt{\eta}} \frac{Y_{e}(1 / 2)}{L}
$$

which means that the limit $\eta \rightarrow 0$ is singular for this first mode. The same phenomenon happens for all odd modes.

In dimensional form (i.e. $\Omega=\omega \sqrt{E I /(\rho h b)} / L^{2}$ ) we have the following expression for the 

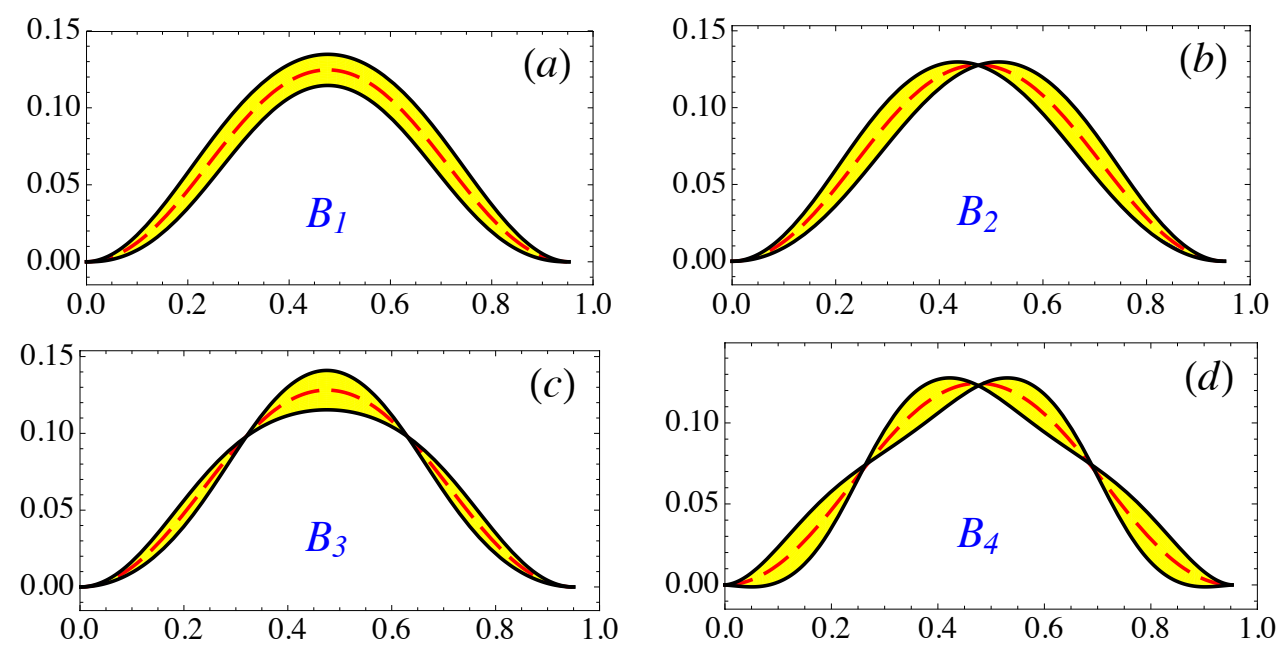

Figure 5: First four modes at $d=0.05$, for $\eta=1 / 4800$ : configurations $D_{1}$ (a), $D_{2}$ (b), $D_{3}$ (c), and $D_{4}$ (d) in Figure 3.
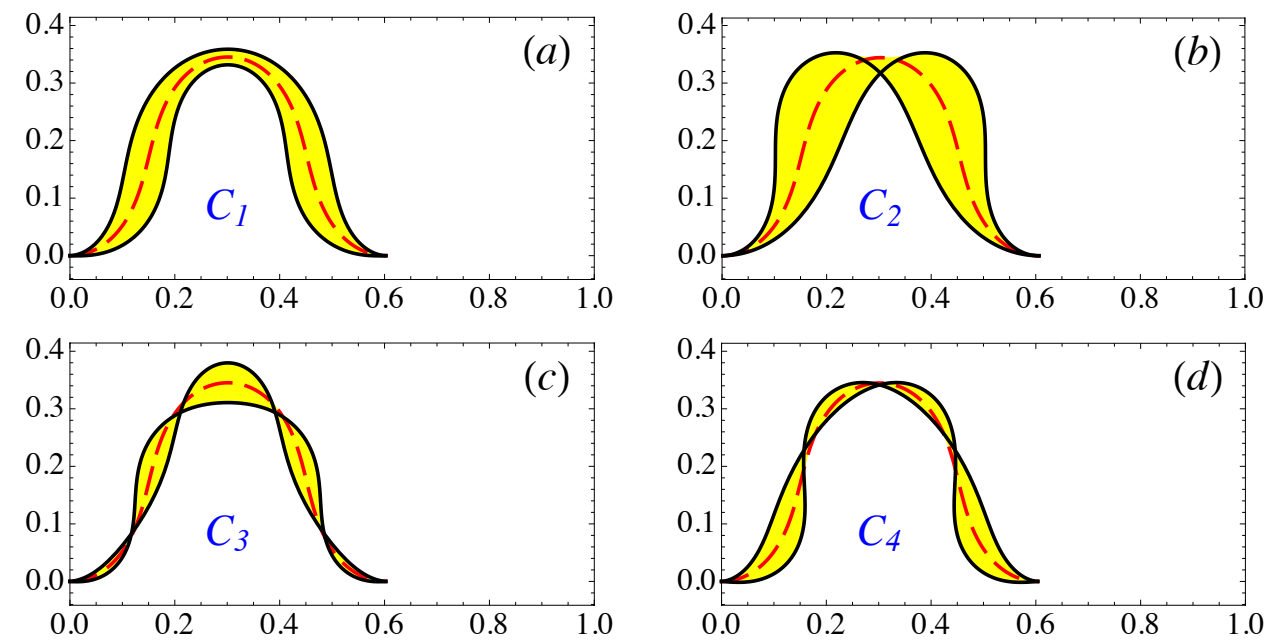

Figure 6: First four modes at $d=0.4$, for $\eta=1 / 4800$ : configurations $E_{1}(\mathrm{a}), E_{2}(\mathrm{~b}), E_{3}$ (c), and $E_{4}$ (d) in Figure 3.

frequency $\Omega$ (in $\mathrm{rad} / \mathrm{s})$ :

$$
\Omega \simeq 8.1 \frac{Y_{e}(L / 2)}{L^{2}} \sqrt{\frac{E}{\rho}}, \quad \text { for } Y_{e}(L / 2) \lesssim 2 h .
$$

The presence of the celerity $c=\sqrt{E / \rho}$ of compression elastic waves in this expression shows that directly after buckling and for a short loading interval (i.e. $Y_{e}(L / 2)=0$ to $Y_{e}(L / 2) \simeq 2 h$, the lowest mode of vibration of a buckled rod is of extension-compression type. We also see in Fig. 7-(b) that for $Y_{e}(L / 2) \gtrsim 8 h$ the behavior is of flexural type (i.e. curves have reached the inextensible asymptote). This separation between two different behaviors in the elastic response 



Figure 7: Post-buckled frequency of the mode emerging from $\omega=0$ at buckling (for $\eta>0$ ). (a) From bottom to top: $1 / \eta=1200,4800,19200,120000,480000$. (b) Same data but with horizontal axis rescaled with $h$; The curves nearly collapse on a master curve whose slope at the origin is $\simeq 28$. The dashed curve (red online), whose slope at the origin is $2 \sqrt{2} \pi^{2} \simeq 27.9$ and which is hardly distinguishable from the previous ones, is the first mode solution of (25). (c) Same data as in (a) but with $d=D / L$ on the horizontal axis. (d) Same data but with the horizontal axis rescaled with $\left(4 \pi^{2} \eta\right)$; As in (b) the curves nearly collapse on a master curve. The dashed curve (red online), which is hardly distinguishable from the previous ones, is the first mode solution of (25).

of the rod could be a way to define the notion of a shallow (resp. deep) buckled equilibrium shape: a shallow (resp. deep) equilibrium shape has a vibrational response that is primarily extensional (resp. flexural).

We now focus on the second mode and show that it does not suffer the same singularity as the first one. The mode frequency emerges from a finite value $\omega_{b}=\omega_{b}(\eta)$ at buckling, and we see in Fig. 3 that its variation (with $p$ ) after buckling is much slower than for the first mode. In Figure 8 the variation of the second mode frequency is plotted as a function of $y_{e}(1 / 2)=Y_{e}(L / 2) / L$. We numerically extract the approximation:

$$
\omega \simeq \omega_{b}(\eta)-(9-5400 \eta)\left(\frac{Y_{e}(L / 2)}{L}\right)^{2}
$$

In conclusion, the limit $\eta \rightarrow 0$ is smooth for the second mode (and in fact for all even modes).

An alternative equation is used in (for example) $[12,13,14,15]$ for the vibrations of postbuckled extensible rods. The transverse displacement $Y(S, T)$ is there solution of the equation:

$$
E I Y^{\prime \prime \prime \prime}+\rho h b \ddot{Y}+P Y^{\prime \prime}=0
$$




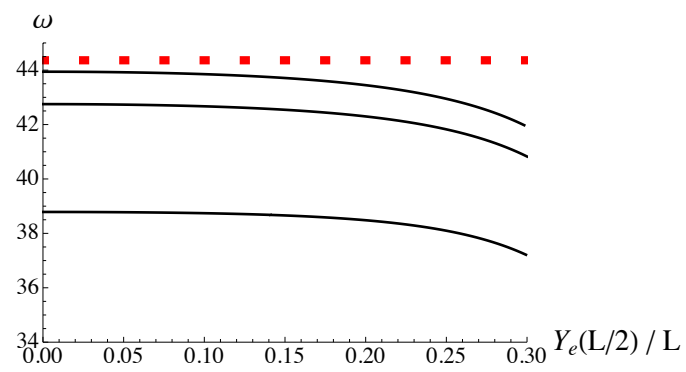

Figure 8: Post-buckled frequency of the second mode (for $\eta>0$ ). Continuous (black)) curves are, from bottom to top, for $1 / \eta=1200,4800,19200$. The (red) dashed curve is the second mode solution of (25).

$$
\text { with } P=\frac{E h b}{L}\left(D-\frac{1}{2} \int_{0}^{L} Y^{\prime 2} d S\right) \text {. }
$$

In this model a certain number of assumptions on the nonlinear terms are made, see e.g. Eq. (B.3)

Calculating the frequency of the first mode just after buckling, we find:

$$
\Omega \simeq \sqrt{2 / 3} \pi^{2} \frac{Y_{e}(L / 2)}{L^{2}} \sqrt{\frac{E}{\rho}}
$$

where $\sqrt{2 / 3} \pi^{2} \simeq 8.06$, in agreement with our result (23). We plot in Fig. 7-(b) and (d) the first mode solution of Eq. (25), and we note that this solution is independent of $\eta$, which is not the case for Kirchhoff equations. Moreover the second mode of (25) is found to be independent of $Y_{e}(L / 2)$ (see Fig. 8), in contradiction with (24). A more comprehensive comparison of the two models is the subject of a forthcoming paper.

\section{Vibrations in the inextensible case}

\section{Vibrations around the straight state}

We consider an inextensible unshearable rod of length $L$, strongly held at both sides by clamps separated by a distance $L$. The rod is held straight and hence no flexural dynamics at all can take place, i.e. flexural vibrations (that would be given by the $\eta=0$ version of Eq. (18)) are impossible here as they would require shortening of the ends.

Axial vibrations are given by:

$$
\begin{aligned}
& \bar{x}^{\prime}(s)=0 \\
& \bar{n}_{x}^{\prime}(s)=-\omega^{2} \bar{x}
\end{aligned}
$$

with boundary conditions $\bar{x}(0)=0=\bar{x}(1)$. The solution is $\bar{x}(s) \equiv 0$, and $\bar{n}_{x}(s) \equiv$ const., where we see that any $\omega$ is admissible. Even if no extensional deformation is present, the load in this statically indeterminate problem can fluctuate with any frequency. This is illustrated in Fig. 9 by the (red) shaded region in the interval $0 \leq p \leq 4 \pi^{2}$. 


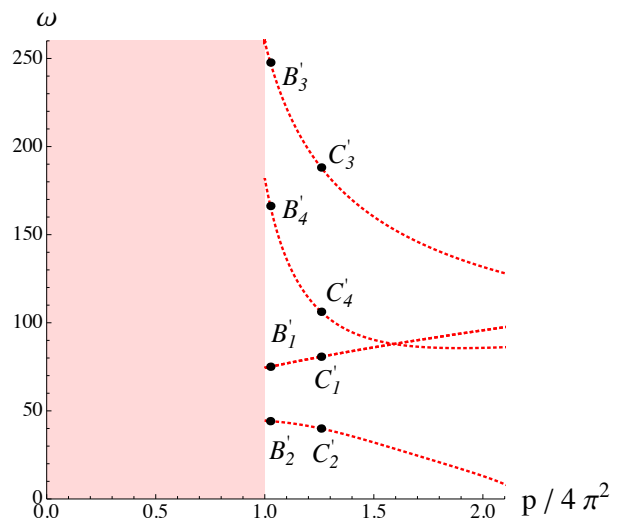

Figure 9: Frequencies for the lowest four vibration modes of a clamped-clamped rod around its fundamental and postbuckled equilibrium configurations in the inextensible case $\eta=0$. The labels $B_{i}^{\prime}, C_{i}^{\prime}$ with $i=1,2,3,4$ correspond to the shapes given in Figs. 10, 11.

Vibrations around the buckled state

As in the extensible case we solve the boundary value problem (13)-(14) numerically with a shooting method. The results are shown in Fig. 9 and in particular we see that at the buckling threshold, the first four frequencies are: $\omega \simeq 44.36,74.4,182.1$, and 259.4. Only one out of two of these frequencies is close to what was found in Fig. 3-(c).

Dynamical shapes $\left(x(s, t)=x_{e}(s)+\bar{x}(s) \cos \omega t, y(s, t)=y_{e}(s)+\bar{y}(s) \cos \omega t\right)$ of the vibrating rod are plotted in Figs. 10, 11.
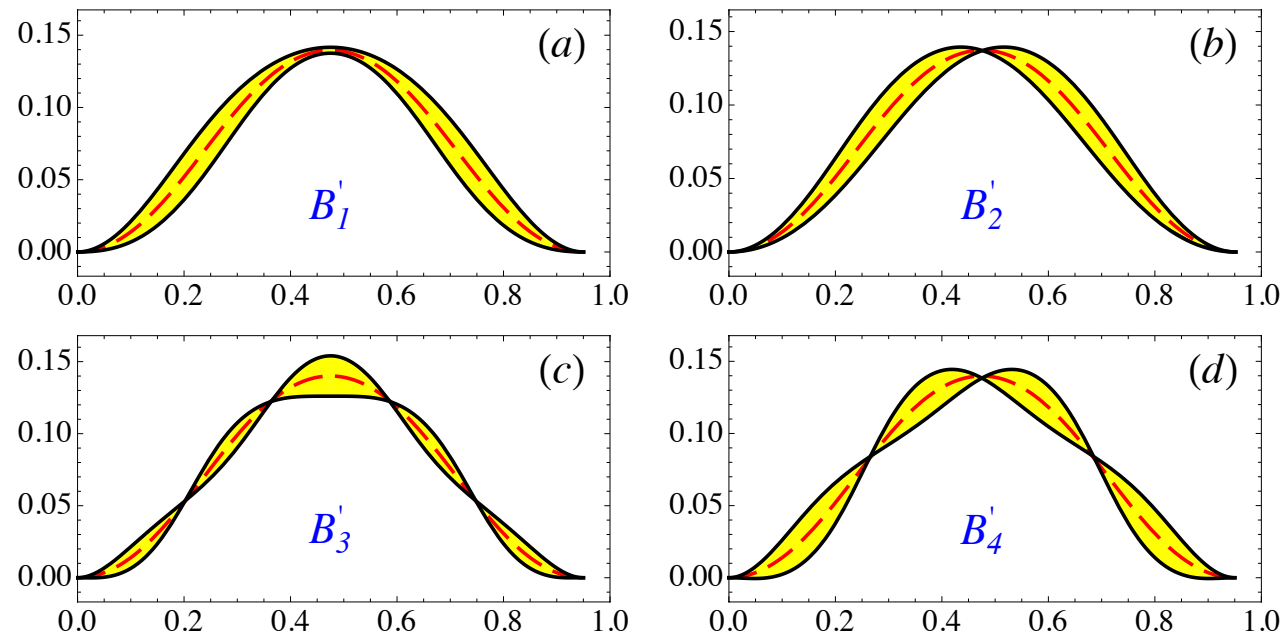

Figure 10: First four modes at $d=0.05$, in the inextensional case: configurations $B_{1}$ (a), $B_{2}$ (b), $B_{3}$ (c), and $B_{4}$ (d) in Figure 3. 

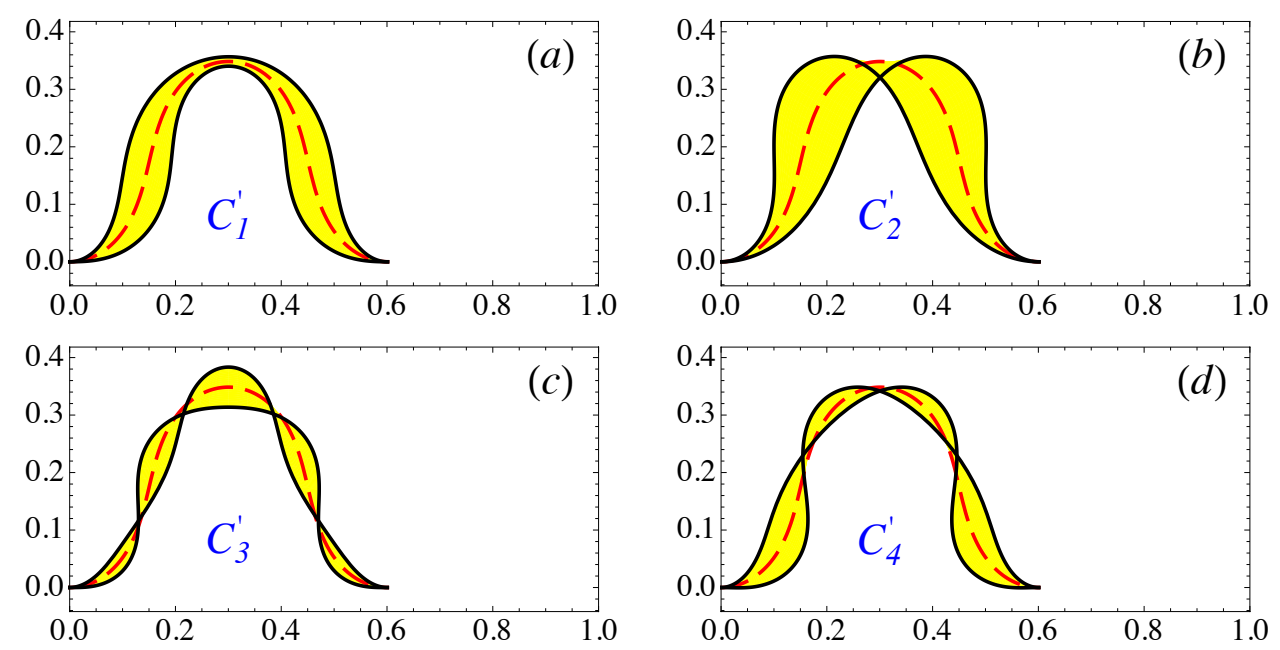

Figure 11: First four modes at $d=0.4$, in the inextensional case: configurations $C_{1}$ (a), $C_{2}$ (b), $C_{3}$ (c), and $C_{4}$ (d) in Figure 3. tensible $(\eta>0)$ and inextensible $(\eta=0)$ cases. On the one hand, we see in Fig. 9 that in the

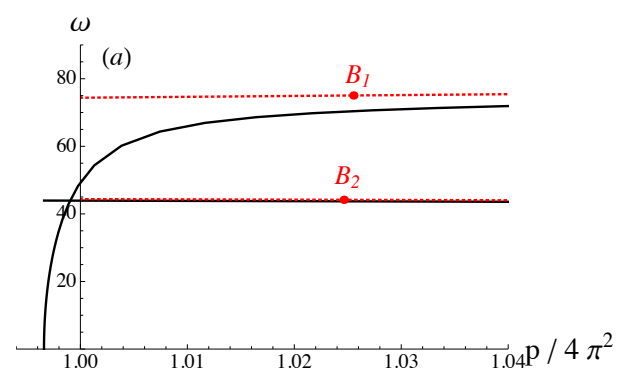

\section{Comparison of the extensible and inextensible results}

We here compare the post-buckled vibrations frequencies obtained numerically for the ex-

Figure 12: Frequencies of a clamped-clamped rod around its post-buckled equilibrium configurations. Comparison of the extensible case ( $\eta=1 / 19200$, plain curve) with the inextensible case (dotted, red) (a) for lowest two vibration modes, and (b) for lowest four vibration modes and plotted as a function of $Y_{e}(L / 2)$, the rise of the buckled equilibrium shape at its middle point. inextensible case there is no curve emerging from $\omega=0$ at buckling, whereas there always is one for all $\eta$ values in the extensible case (Fig. 3). On the other hand, in the inextensible case there is a curve emerging from $\omega \simeq 74.4$, nowhere near any of the extensible curves. We plot in Fig. 12-(a) a comparison of the inextensible $\eta=0$ and extensible $\eta=1 / 19200$ cases, for the first two modes. We see that the second extensible mode (emerging from $\omega \simeq 44$ at buckling) is always very close to its inextensible counterpart, but that the first extensible mode (emerging from $\omega=0$ at buckling) is first very far from its inextensible counterpart and only approaches it later in the post-buckling regime. The consequence is that for a short interval after buckling the 
inextensible model wrongly predicts a fundamental mode at $\omega \simeq 44$ whereas for any 'real life' elastic rod there is a vibration mode with lower frequency.

A full comparison between extensible and inextensible cases shows that all extensible odd modes experiences a similar mismatch with their inextensible counterpart, see Fig. 12-(b) for the first four modes. For the solution at buckling, with $p$ given by (21), we further investigate numerically the variation with $\eta$ of the frequencies of the first four modes in the extensible case and compare them with the first four frequency of the inextensible case $\eta=0$. In Fig. 13, we

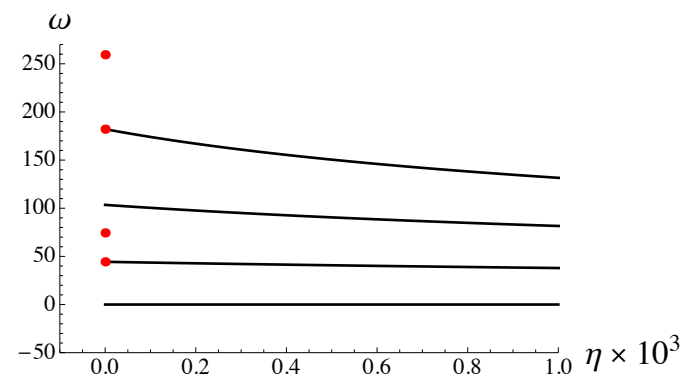

Figure 13: The curves show the variation with $\eta$ of the frequencies of the first four modes in the extensible case, at the buckling threshold. The (red) dots at $\eta=0$ show the frequency values of the first four modes of the inextensible case, at the buckling threshold. A mismatch for half of the modes exists.

\section{Analytical study for the inextensible case}

In order to understand the mismatch of the odd modes' frequencies at buckling between the extensible and inextensible cases, we look at the problem analytically. In the inextensible case, buckling happens for $p=4 \pi^{2}$. We look for the frequencies that emerge from the continuum present for $p \leq 4 \pi^{2}$. If the $\eta \rightarrow 0$ limit was not singular we would just look for the solutions of (20) for $\eta=0$ and $p=4 \pi^{2}$, that is:

$$
2 k^{+} k^{-}\left(\cos k^{+} \cosh k^{-}-1\right)+4 \pi^{2} \sin k^{+} \sinh k^{-}
$$

with $k^{ \pm}=\sqrt{\sqrt{4 \pi^{4}+\omega^{2}} \pm 2 \pi^{2}}$. The solutions are listed in Table 1 and we see that, for the odd modes, they do not match what is found numerically.

To investigate matter further we set $\eta=0$ in Eqs. (10), and look for the frequency values that exist just after buckling (i.e. around post-buckled configurations). In the post-buckled configuration, the equilibrium equations and the boundary conditions are given by

$$
\begin{aligned}
& n_{y e}=\text { const. } \\
& n_{x e}=-p \\
& \theta_{e}^{\prime \prime}=-p \sin \theta_{e}-n_{y e} \cos \theta_{e} \text { with } \theta_{e}(0)=0=\theta_{e}(1) \\
& x_{e}^{\prime}=\cos \theta_{e} \quad \text { with } x_{e}(1)-x_{e}(0)=1-d \\
& y_{e}^{\prime}=\sin \theta_{e} \quad \text { with } y_{e}(0)=y_{e}(1) \text {, } \\
& 14
\end{aligned}
$$




\begin{tabular}{|l|c|c|c|c|c|c|c|c|}
\hline$i$ & 1 & 2 & 3 & 4 & 5 & 6 & 7 & 8 \\
\hline$\omega$ & 0 & 44.36 & 103.5 & 182.1 & 280.6 & 398.8 & 536.8 & 694.6 \\
\hline$k^{+}$ & $2 \pi$ & 8.26 & 11.18 & 14.25 & 17.35 & 20.5 & 23.6 & 26.7 \\
\hline$\left(k^{+} \bmod 2 \pi\right) /(\pi / 2)$ & & 1.26 & 3.12 & 1.07 & 3.04 & 1.03 & 3.02 & 1.02 \\
\hline$k^{-}$ & 0 & 5.37 & 9.25 & 12.8 & 16.2 & 19.5 & 22.7 & 25.98 \\
\hline
\end{tabular}

Table 1: Frequencies and wave numbers for the lowest eight modes of vibration as given by Eq. (20) for $p=4 \pi^{2}$ and $\eta=0$. This also corresponds to the first eight solutions of $P_{1}\left(\omega_{0}\right)=0$ (see Eq. (38)).

and without loss of generality, we choose $x_{e}(0)=y_{e}(0)=0$. For equilibrium modes whose shapes are invariant when reflected along the line parallel to the $\boldsymbol{e}_{\boldsymbol{y}}$ axis and containing the point $(x(1 / 2), 0)$, we have $n_{y e}=$ const. $=0$ in Eq. (29a). The first bifurcated equilibrium mode, represented in Fig. 1 and on which we focus, is such a mode. We address the behavior of the solutions after but close to buckling. Therefore, we expand the variables $\theta_{e}(s)$ and $y_{e}(s)$ in powers of $\epsilon$, a small parameter measuring the distance from buckling:

$$
\begin{aligned}
\theta_{e}(s) & =\epsilon \theta_{1}(s)+\epsilon^{2} \theta_{2}(s)+\epsilon^{3} \theta_{3}(s)+O\left(\epsilon^{4}\right) \\
x_{e}(s) & =\epsilon x_{1}(s)+\epsilon^{2} x_{2}(s)+\epsilon^{3} x_{3}(s)+O\left(\epsilon^{4}\right) \\
y_{e}(s) & =\epsilon y_{1}(s)+\epsilon^{2} y_{2}(s)+\epsilon^{3} y_{3}(s)+O\left(\epsilon^{4}\right) \\
p & =p_{0}+\epsilon p_{1}+\epsilon^{2} p_{2}+\epsilon^{3} p_{3}+O\left(\epsilon^{4}\right)
\end{aligned}
$$

We substitute these expansions in the equilibrium equations (29), which have to be satisfied to all orders in $\epsilon$. The solution up to order 3 reads:

$$
\begin{aligned}
\theta_{e}(s) & =\epsilon \sin 2 \pi s+\frac{\epsilon^{3}}{48} \cos ^{2}(2 \pi s) \sin (2 \pi s)+O\left(\epsilon^{4}\right) \\
x_{e}(s) & =s+\frac{\epsilon^{2}}{16 \pi}(\sin 4 \pi s-4 \pi s)+O\left(\epsilon^{4}\right) \\
y_{e}(s) & =\frac{\epsilon}{2 \pi}(1-\cos 2 \pi s)+\frac{\epsilon^{3}}{384 \pi}(-20+23 \cos (2 \pi s)-3 \cos (6 \pi s))+O\left(\epsilon^{4}\right) \\
p & =4 \pi^{2}+\epsilon^{2} \pi^{2} / 2+O\left(\epsilon^{4}\right)
\end{aligned}
$$

In order to relate $\epsilon$ to the control parameter $d$ and the amplitude after bifurcation, we compute the end-shortening

$$
d=1-\left(x_{e}(1)-x_{e}(0)\right)=\epsilon^{2} / 4+O\left(\epsilon^{4}\right)=2\left(\frac{p}{4 \pi^{2}}-1\right)+O\left(\epsilon^{4}\right),
$$

and the rod maximum deflection

$$
y_{e}(1 / 2)=\frac{\epsilon}{\pi}\left(1-\frac{5}{48} \epsilon^{2}\right)+O\left(\epsilon^{4}\right) .
$$

\section{Vibration around the post-buckled equilibrium}

We expand all modal variables $\left(\bar{x}, \bar{y}, \bar{\theta}, \bar{m}, \bar{n}_{x}, \bar{n}_{y}\right)$ and the frequency $\omega$ in powers of $\epsilon$. For instance, we have $\omega=\omega_{0}+\epsilon \omega_{1}+\epsilon^{2} \omega_{2}+\epsilon^{3} \omega_{3}+O\left(\epsilon^{4}\right)$, and so on. We can now solve equations 


$$
\begin{aligned}
\bar{x}_{0}^{\prime} & =0 \text { with } \bar{x}_{0}(0)=0=\bar{x}_{0}(1) \\
\bar{n}_{x 0}^{\prime} & =-\omega_{0}^{2} \bar{x}_{0} \\
\bar{y}_{0}^{\prime \prime \prime}+4 \pi^{2} \bar{y}_{0}^{\prime \prime}-\omega_{0}^{2} \bar{y}_{0} & =0 \text { with } \bar{y}_{0}(0)=\bar{y}_{0}(1)=\bar{y}_{0}^{\prime}(0)=\bar{y}_{0}^{\prime}(1)=0
\end{aligned}
$$

259

The first two equations describe the longitudinal mode and are decoupled from the third one which is associated with the transverse mode. More precisely, the longitudinal mode is given by

$$
\bar{x}_{0}(s)=0 \text { and } \bar{n}_{x 0}(s) \text { constant } .
$$

whereas for the transverse mode, the solution $\bar{y}_{0}(s)$ is

$$
\bar{y}_{0}(s)=A_{0}\left(\frac{\cos k_{0}^{+} s-\cosh k_{0}^{-} s}{\cos k_{0}^{+}-\cosh k_{0}^{-}}-\frac{k_{0}^{-} \sin k_{0}^{+} s-k_{0}^{+} \sinh k_{0}^{-} s}{k_{0}^{-} \sin k_{0}^{+}-k_{0}^{+} \sinh k_{0}^{-}}\right),
$$

262 with $k_{0}^{ \pm} \stackrel{\text { def }}{=} \sqrt{\sqrt{4 \pi^{4}+\omega_{0}^{2}} \pm 2 \pi^{2}}$. The boundary conditions impose that

$$
\begin{aligned}
A_{0} P_{1}\left(\omega_{0}\right) & =0 \text { with } \\
P_{1}\left(\omega_{0}\right) & \stackrel{\text { def }}{=} 2 k_{0}^{+} k_{0}^{-}\left(\cos k_{0}^{+} \cosh k_{0}^{-}-1\right)+4 \pi^{2} \sin k_{0}^{+} \sinh k_{0}^{-}
\end{aligned}
$$

which is an equation for $\omega_{0}$. The first eight solutions of $P_{1}\left(\omega_{0}\right)=0$ are listed in Table 1 . Note that to order $\epsilon^{0}$ there is no frequency jump. To order $\epsilon^{1}$ we have

$$
\begin{aligned}
\bar{x}_{1}^{\prime}= & \bar{\theta}_{0} \sin 2 \pi s \text { with } \bar{x}_{1}(0)=0=\bar{x}_{1}(1) \\
\bar{n}_{x 1}^{\prime}= & -\omega_{0}^{2} \bar{x}_{1}-2 \omega_{0} \omega_{1} \bar{x}_{0} \\
\bar{y}_{1}^{\prime \prime \prime \prime}+4 \pi^{2} \bar{y}_{1}^{\prime \prime}-\omega_{0}^{2} \bar{y}_{1}= & 2 \omega_{0} \omega_{1} \bar{y}_{0}+2 \pi \bar{n}_{x 0} \cos 2 \pi s \\
& \text { with } \bar{y}_{1}(0)=\bar{y}_{1}(1)=\bar{y}_{1}^{\prime}(0)=\bar{y}_{1}^{\prime}(1)=0 .
\end{aligned}
$$

We start by solving equation (39a). The boundary condition $\bar{x}_{1}(1)=0$ implies

$$
\begin{aligned}
A_{0} P_{2}\left(\omega_{0}\right)= & 0 \text { with } \\
P_{2}\left(\omega_{0}\right) \stackrel{\text { def }}{=} & k_{0}^{+} k_{0}^{-}\left(\left(k_{0}^{+2}-2 \pi^{2}\right)\left(\cos k_{0}^{+}-\cosh k_{0}^{-}\right)+2 \pi^{2}\left(\cos k_{0}^{+} \cosh k_{0}^{-}-1\right)\right) \\
& +\left(k_{0}^{+2} k_{0}^{-2}+8 \pi^{4}\right) \sin k_{0}^{+} \sinh k_{0}^{-} .
\end{aligned}
$$

The solutions have to satisfy Eqs. (37) and (40), which are transcendental equations for $\omega_{0}$. A numerical root finding analysis reveals that $P_{1}\left(\omega_{0}\right)=0$ and $P_{2}\left(\omega_{0}\right)=0$ share half of their roots, see Table 2 where columns with an even index correspond to common roots and match numerical values at $p=4 \pi^{2}$ for the continuous curves plotted in Fig. 3-(d). In the case of a common root, Eqs. (37) and (40) are fulfilled for non-vanishing $A_{0}$, and the corresponding modes have frequencies that are continuous in the control parameter close to buckling.

In the case of distinct roots, we are compelled to set $A_{0}=0$. In this case where $P_{1}\left(\omega_{0}\right) \neq 0$, we solve Eqs. (39a) and (39b) to obtain $\bar{x}_{1}(s)=0$ and $\bar{n}_{x 1}(s)$ constant. The general solution of Eqs. (39c), (39d) is then

$$
\begin{aligned}
\bar{y}_{1}(s)= & \frac{2 \pi \bar{n}_{x 0}}{k_{0}^{+2} k_{0}^{-2} P_{1}\left(\omega_{0}\right)}\left[c_{1} k_{0}^{+} \cos k_{0}^{+} s-c_{1} k_{0}^{+} \cosh k_{0}^{-} s-c_{2} k_{0}^{-} \sin k_{0}^{+} s+c_{2} k_{0}^{+} \sinh k_{0}^{-} s\right. \\
& \left.+P_{1}\left(\omega_{0}\right)\left(\cos k_{0}^{+} s-\cos 2 \pi s\right)\right] \\
& 16
\end{aligned}
$$




\begin{tabular}{|l|c|c|c|c|c|c|c|c|}
\hline$i$ & 1 & 2 & 3 & 4 & 5 & 6 & 7 & 8 \\
\hline$\omega_{0}$ & 0 & 44.36 & 169.4 & 182.1 & 390.6 & 398.8 & 688.5 & 694.6 \\
\hline$k_{0}^{+}$ & $2 \pi$ & 8.26 & 13.8 & 14.25 & 20.3 & 20.5 & 26.6 & 26.7 \\
\hline$\left(k_{0}^{+} \bmod 2 \pi\right) /(\pi / 2)$ & & 1.26 & 0.78 & 1.07 & 0.90 & 1.03 & 0.95 & 1.02 \\
\hline$k_{0}^{-}$ & 0 & 5.37 & 12.3 & 12.8 & 19.3 & 19.5 & 25.8 & 25.98 \\
\hline
\end{tabular}

Table 2: First eight solutions of $P_{2}\left(\omega_{0}\right)=0$ (see Eq. (41)).

275

where

$$
\begin{aligned}
& c_{1}=k_{0}^{-}\left(\cos k_{0}^{+}-1\right)\left(\cosh k_{0}^{-}+1\right)+k_{0}^{+} \sin k_{0}^{+} \sinh k_{0}^{-} \\
& c_{2}=k_{0}^{+} \sin k_{0}^{+}\left(\cosh k_{0}^{-}-1\right)+k_{0}^{-}\left(\cos k_{0}^{+}-1\right) \sinh k_{0}^{-}
\end{aligned}
$$

In order to select a mode, we need to proceed to order $\epsilon^{2}$, which reads

$$
\bar{x}_{2}^{\prime}(s)=-\bar{y}_{1}^{\prime}(s) \sin 2 \pi s \text { with } \bar{x}_{2}(0)=0=\bar{x}_{2}(1)
$$

277 The boundary conditions at $s=1$ impose $P_{3}\left(\omega_{0}\right) / P_{1}\left(\omega_{0}\right)=0$ where

$$
\begin{aligned}
P_{3}\left(\omega_{0}\right)= & 2 k_{0}^{-2} k_{0}^{+2}\left(\cosh k_{0}^{-} \cos k_{0}^{+}-1\right)-4 k_{0}^{+}\left(k_{0}^{-2}+k_{0}^{+2}\right) \sin k_{0}^{+}\left(\cosh k_{0}^{-}-1\right) \\
& +\left(4 k_{0}^{-}\left(k_{0}^{-2}+k_{0}^{+2}\right)\left(1-\cos k_{0}^{+}\right)+4 \pi^{2} k_{0}^{-} k_{0}^{+} \sin k_{0}^{+}\right) \sinh k_{0}^{-}
\end{aligned}
$$

Oddly enough this function $P_{3}\left(\omega_{0}\right)$ has the same set of common roots as $P_{1}\left(\omega_{0}\right)$ and $P_{2}\left(\omega_{0}\right)$, see Table 3. The other roots correspond to the frequency values at which the (discontinuous) odd mode curves emerge from $p=4 \pi^{2}$ in Fig. 3-(d). Moreover one can verify that

$$
P_{3}\left(\omega_{0}\right)=k_{0}^{+} k_{0}^{-} P_{1}\left(\omega_{0}\right)-8\left(k_{0}^{+2}+k_{0}^{-2}\right)\left(k_{0}^{+} \cos \frac{k_{0}^{+}}{2} \tanh \frac{k_{0}^{-}}{2}-k_{0}^{-} \sin \frac{k_{0}^{+}}{2}\right) \sinh k_{0}^{-} \sin \frac{k_{0}^{+}}{2}
$$

which implies that the common roots must verify:

$$
\frac{k_{0}^{+}}{2} \tanh \frac{k_{0}^{-}}{2}=\frac{k_{0}^{-}}{2} \tan \frac{k_{0}^{+}}{2}
$$

These roots correspond to frequencies that do not vary abruptly after buckling has occurred. An approximate formula is $k_{0}^{+} \simeq \pi / 2+2 j \pi$ (with positive integers $j$ ), which yields $\omega_{0} \simeq(\pi / 2)^{2}(4 j+$ 1) $\sqrt{(4 j-3)(4 j+5)}$ (corresponding to columns with $i>1$ even in Table 3 ).

Formulas for the roots of the three functions $P_{1}, P_{2}$, and $P_{3}$ in the limit of large $k_{0}^{+}$are given in Appendix C. In particular it is shown that the set of roots of $P_{3}$ which is not in common with $P_{1}$ and $P_{2}$ is such that $k_{0}^{+} \simeq 3 \pi / 2+2 j \pi$ (with positive integers $j$ ). This implies that the frequencies emerging from buckling are such that $\omega_{0} \simeq(\pi / 2)^{2}(4 j+3) \sqrt{(4 j+7)(4 j-1)}$ (corresponding to columns with $i>1$ odd in Table 3 ).

\section{Discussion}

In [11] it is shown that, under certain hypotheses, in the zero thickness limit a slender cylinder behaves either as a flexible rod or as an extensible string and that one does not need to consider 


\begin{tabular}{|l|c|c|c|c|c|c|c|c|}
\hline$i$ & 1 & 2 & 3 & 4 & 5 & 6 & 7 & 8 \\
\hline$\omega_{0}$ & 0 & 44.36 & 74.4 & 182.1 & 259.4 & 398.8 & 517.4 & 694.6 \\
\hline$k_{0}^{+}$ & $2 \pi$ & 8.26 & 9.83 & 14.25 & 16.7 & 20.5 & 23.2 & 26.7 \\
\hline$\left(k_{0}^{+} \bmod 2 \pi\right) /(\pi / 2)$ & & 1.26 & 2.26 & 1.07 & 2.65 & 1.03 & 2.76 & 1.02 \\
\hline$k_{0}^{-}$ & 0 & 5.37 & 7.56 & 12.8 & 15.5 & 19.5 & 22.3 & 25.98 \\
\hline
\end{tabular}

Table 3: First eight solutions of $P_{3}\left(\omega_{0}\right)=0$ (see Eq. (46)).

both bending and extension in a slender rod problem. Marigo and Meunier [11] result was obtain for the statics of a clamped-free rod subjected to body and concentrated forces. We have showed here that for displacement control clamped-clamped boundary conditions a model including both bending and extension is necessary to correctly describe the vibrations of the rod. One could then ask which constraint should be relaxed in order to fall back into the results of [11]. We simply remark that in the load control case, see Appendix D, the inextensible limit is smooth and hence a model including bending alone correctly captures the vibrational behavior of the rod.

From a theoretical point of view, the singular inextensible limit in the displacement control case is rather surprising and unexpected. Indeed, Equations (13) can be easily recast in the form of a classical eigenvalue problem

$$
\mathcal{L} \mathbf{X}=\omega^{2} \mathbf{M} \mathbf{X}
$$

where $\mathbf{X}$ is the six-dimensional vector build from the six normal mode variables, $\mathcal{L}$ is a firstorder linear operator in $L^{2}([0,1])$, (the set of square integrable functions on the unit interval) and $\mathbf{M}$ is an inertia matrix. Therefore, a naive application of the classical theory of perturbation of eigenvalues for linear operators would suggest that once the eigenvalues have been found for a value of the parameter $\eta$, they can be locally continued in this parameter. That is we would expect the curves $\omega(p)$ of Fig. 3 to vary continuously as the parameter $\eta$ is decreased to zero. While it is true for some curves, other curves behaves singularly in the $\eta \rightarrow 0$ limit. The fundamental mathematical reason for this phenomenon is that both the null spaces of the inertia matrix $\mathbf{M}$ and the linear operator $\mathcal{L}$ have a non-empty intersection in the inextensible $(\eta=0)$ case. Mathematically, the classical theory does not apply and new conditions for the analytic continuation of frequencies with respect to the parameters emerge. Some frequencies satisfy these relations (and hence can be analytically continued) while others do not. Finally, also due to the joint degeneracy of $\mathbf{M}$ and $\mathcal{L}$, there exists for $\eta=0$ a continuous family of solutions at the critical load, given by an arbitrary increase along $\bar{n}_{x}$ (i.e. solutions of Eq. (27)). For larger values of the load but still at $\eta=0$, some of these solutions are selected and emerge, apparently out of the blue. The mathematical structure of these linear problems and how they are related to various limits (nearly inextensible rods) deserves further attention.

A tentative classification for the modes is given by the number of nodes present in $0<s<1$, a mode $i$ having $i-1$ nodes and $i$ antinodes. We see that this classification works for the modes in the pre-buckling regime (Fig. 4), but fails for the third mode in the inextensible $(\eta=0)$ case (see shape $B_{3}^{\prime}$ in Fig. 10 and $C_{3}^{\prime}$ in Fig. 11), as well as in the extensible rod $(\eta>0)$ case provided we are far enough in the post-buckling regime (see shape $C_{3}$ in Fig. 6).

An interesting situation arises when the first mode intersects with the second mode. This can easily be obtained by computing an approximation of the buckled rod rise $Y_{e}(L / 2)$ for which the first mode frequency $\omega^{(1)} \simeq 28 Y_{e}(L / 2) / h$ meets the second mode frequency $\omega^{(2)} \simeq 44$. This happens for $Y_{e}(L / 2) \simeq 1.6 h$. At this height the shape of the fundamental mode changes from a 
single bump wave to a double bump wave. Anecdotically, we note that piano soundboards are precisely tuned in this parameter range (e.g. $h=1 \mathrm{~cm}, L=2 \mathrm{~m}$, and $Y_{e}(L / 2)=1 \mathrm{~cm}$ ). To which extent this toy model is relevant for the real problem of the piano soundboard and whether piano manufacturers are using the distinction between shallow shapes (where extension prevails) and deep buckled shapes (where bending prevails) to enrich the sound is a tantalizing idea that deserves further attention. We also note that in the case of the piano, the soundboard is a plate and not a rod, and that furthermore the soundboard is linked to the piano strings by a bridge, so really the whole system should be studied.

\section{Conclusion}

We have studied the in-plane vibrations of a slender extensible and shearable elastic rod around its post-buckled equilibrium configuration, in the displacement control case. We have shown that after buckling there is a narrow window in the loading parameter values in which half of the vibration frequencies vary abruptly. As the thickness of the rod is decreased, the vibration frequencies tend toward limiting values which do not correspond to what is found with a fully inextensible, unshearable model (i.e. an elastica). This mismatch present for half of the vibration frequencies has been found numerically and proved analytically. We conclude that we have identified a loading setup where the elastica model for slender rods fails to give the correct answer.

\section{Acknowledgements}

It is a pleasure to thank Olivier Thomas for discussions on Eq. (25). This publication is based in part upon work supported by Award No. KUK-C1-013-04, made by King Abdullah University of Science and Technology (KAUST) (AG). AG is a Wolfson/Royal Society Merit Award holder.

\section{Appendix A. Closed form solution for the planar elastica}

In the inextensible, unshearable case, the solution of system (10) with $\ddot{x}_{e}=0$ and $\ddot{y}_{e}=0$ corresponds to the equilibrium of the planar elastica, first studied by Euler [16] (see [17] for an historical account).

Closed-form solutions of these equations can be written in terms of elliptic functions, see e.g. [18]. In particular the dotted (red) curve in Fig. 2 has the parametric expression:

$$
\begin{aligned}
& p=16 K(\lambda)^{2} \\
& d=2\left(1-\frac{E(\lambda)}{K(\lambda)}\right)^{2}
\end{aligned}
$$

357 with $\lambda \in[0,1)$. The elliptic integrals are defined as $K(\lambda)=\int_{0}^{\pi / 2}\left(1-\lambda \sin ^{2} \theta\right)^{-1 / 2} d \theta$ and $E(\lambda)=$ ${ }_{358} \int_{0}^{\pi / 2}\left(1-\lambda \sin ^{2} \theta\right)^{1 / 2} d \theta$. Developing Eqs. (A.1) and (A.2) for $\lambda \ll 1$ yields Eqs. (31d) and (32) 359 with $\epsilon^{2}=4 \lambda$. 


\section{Appendix B. Von Karman kinematics and 'Strength of materials' notations}

In section 2, we have introduced the Cosserat-Kirchhoff notations where the normal force $N_{1}$ is related to the extension $e_{1}$ through the constitutive law (6) and where the current position $(X, Y)$ of the central axis of the rod is given by Eqs. (8a) and (8b), all these quantities being functions of the arc-length $S$ of the reference configuration $\left(X_{\text {ref }}, Y_{\text {ref }}\right)=(S, 0)$. In 'Strength of materials' notations one uses the displacements (see Fig.1):

$$
U=X-S, \quad V=Y,
$$

also functions of the arc-length $S$ of the reference configuration. In the current configuration the derivative of the current position with regard to $S$ will not yield a unit vector if extension occurs. If we restrict to the case where shear is not present (i.e. $e_{2}=0$ ), we have $X^{\prime}(S)^{2}+Y^{\prime}(S)^{2}=$ $\left(1+e_{1}\right)^{2}$ which yields:

$$
U^{\prime}+\frac{1}{2} V^{\prime 2}-e_{1}=\frac{1}{2}\left(e_{1}^{2}-U^{\prime 2}\right)
$$

Usually the right-hand side is neglected and the following von Karman approximation is used:

$$
U^{\prime}+\frac{1}{2} V^{\prime 2}-e_{1} \simeq 0
$$

\section{Appendix C. Analytical formulas for the roots of the functions $P_{1}, P_{2}$, and $P_{3}$}

In the limit of large $k_{0}^{+}$, that is in the limit of large $k_{0}^{-}=\sqrt{k_{0}^{+2}-4 \pi^{2}}$ and high frequencies $\omega_{0}=k_{0}^{+} k_{0}^{-}$, we have:

$$
k_{0}^{-} \simeq k_{0}^{+}-\frac{2 \pi^{2}}{k_{0}^{+}}-\frac{2 \pi^{4}}{k_{0}^{+3}}+O\left(\frac{1}{k_{0}^{+5}}\right) .
$$

Function $P_{1}\left(\omega_{0}\right)$ (see Eq. (38)) then reads:

$$
P_{1}\left(\omega_{0}\right) \simeq \mathrm{e}^{k_{0}^{-}}\left[k_{0}^{+2} \cos k_{0}^{+}+2 \pi^{2}\left(\sin k_{0}^{+}-\cos k_{0}^{+}\right)\right],
$$

and the solutions to $P_{1}\left(\omega_{0}\right)=0$, for large $k_{0}^{+}$, are:

$$
\begin{aligned}
& k_{0}^{+} \simeq \frac{\pi}{2}+\frac{1}{2 j^{2}}+2 j \pi \quad \text { with } i=2 j \\
& k_{0}^{+} \simeq \frac{3 \pi}{2}+\frac{1}{2 j^{2}}+2 j \pi \quad \text { with } i=2 j+1
\end{aligned}
$$

where $j$ is a large integer, and $i$ is the mode number (i.e. column number in Table 1 ). In the same limit of high frequencies, function $P_{2}\left(\omega_{0}\right)$ (see Eq. (41)) reads:

$$
P_{2}\left(\omega_{0}\right) \simeq \frac{1}{2} \mathrm{e}^{k_{0}^{-}}\left[k_{0}^{+4}\left(\sin k_{0}^{+}-1\right)+2 \pi^{2} k_{0}^{+2} \cos k_{0}^{+}+6 \pi^{4}\right],
$$

$$
\begin{aligned}
& k_{0}^{+} \simeq \frac{\pi}{2}-\frac{3}{2 j^{2}}+2 j \pi \quad \text { with } i=2 j-1 \\
& k_{0}^{+} \simeq \frac{\pi}{2}+\frac{1}{2 j^{2}}+2 j \pi \quad \text { with } i=2 j
\end{aligned}
$$


where $j$ is a large integer, and $i$ is the mode number (i.e. column number in Table 2). In the same limit of high frequencies, function $P_{3}\left(\omega_{0}\right)$ (see Eq. (46)) reads:

$$
P_{3}\left(\omega_{0}\right) \simeq \mathrm{e}^{k_{0}^{-}}\left[4 k_{0}^{+3}\left(1-\sin k_{0}^{+}\right)+k_{0}^{+4} \cos k_{0}^{+}+2 \pi^{2} k_{0}^{+2}\right],
$$

and the solutions to $P_{3}\left(\omega_{0}\right)=0$, for large $k_{0}^{+}$, are:

$$
\begin{aligned}
& k_{0}^{+} \simeq \frac{\pi}{2}+\frac{1}{2 j^{2}}+2 j \pi \text { with } i=2 j \\
& k_{0}^{+} \simeq \frac{3 \pi}{2}-\frac{4}{\pi j}+2 j \pi \quad \text { with } i=2 j+1
\end{aligned}
$$

where $j$ is a large integer, and $i$ is the mode number (i.e. column number in Table 3 ). We see that in this limit the three functions $P_{1}, P_{2}$, and $P_{3}$ share half of their roots, namely those given by (C.3), (C.7), or (C.9).

\section{Appendix D. Smooth inextensible limit in the load control case}

We here show that in the case where the axial load $p$ is controlled, the inextensible limit $(\eta \rightarrow 0)$ is no longer singular. The boundary conditions for this case read:

$$
\begin{array}{ll}
x(0, t)=0 & n_{x}(1, t)=-p, \\
y(0, t)=0 & y(1, t)=0, \\
\theta(0, t)=0 & \theta(1, t)=0 .
\end{array}
$$

Vibrations of an inextensible rod are then possible before buckling as the right end is allowed to move axially. We see in Fig. D.14 that the inextensible case $\eta=0$ is obtain as the smooth limit $\eta \rightarrow 0$ of extensible case, and that no mismatch is present (in particular there is a mode starting from $\omega=0$ after buckling in the inextensible case).

\section{References}

[1] L. N. Virgin, Vibration of axially loaded structures, Cambridge University Press, 2007.

[2] C. Touzé, O. Thomas, A. Chaigne, Hardening/softening behaviour in non-linear oscillations of structural systems using non-linear normal modes, Journal of Sound and Vibration 273 (2004) 77 - 101.

[3] N. C. Perkins, Planar vibration of an elastica arch: Theory and experiment, Journal of Vibration and Acoustics 112 (1990) 374-379.

[4] S. T. Santillan, L. N. Virgin, R. H. Plaut, Post-buckling and vibration of heavy beam on horizontal or inclined rigid foundation, Journal of Applied Mechanics 73 (2006) 664-671.

[5] R. H. Plaut, L. N. Virgin, Vibration and snap-through of bent elastica strips subjected to end rotations, Journal of Applied Mechanics 76 (2009) 041011.

[6] P. H. Bilhuber, C. A. Johnson, The influence of the soundboard on piano tone quality, The Journal of the Acoustical Society of America 11 (1940) 311-320.

[7] A. Mamou-Mani, J. Frelat, C. Besnainou, Numerical simulation of a piano soundboard under downbearing, The Journal of the Acoustical Society of America 123 (2008) 2401-2406.

[8] A. Mamou-Mani, J. Frelat, C. Besnainou, Prestressed soundboards: Analytical approach using simple systems including geometric nonlinearity, Acta Acustica united with Acustica 95 (2009) 915-928.

[9] A. Chaigne, C. Touzé, O. Thomas, Nonlinear vibrations and chaos in gongs and cymbals, Acoustical Science and Technology 26 (2005) 403-409.

[10] S. S. Antman, Nonlinear problems of elasticity, Springer-Verlag, New York, 2nd edition, 2004. 


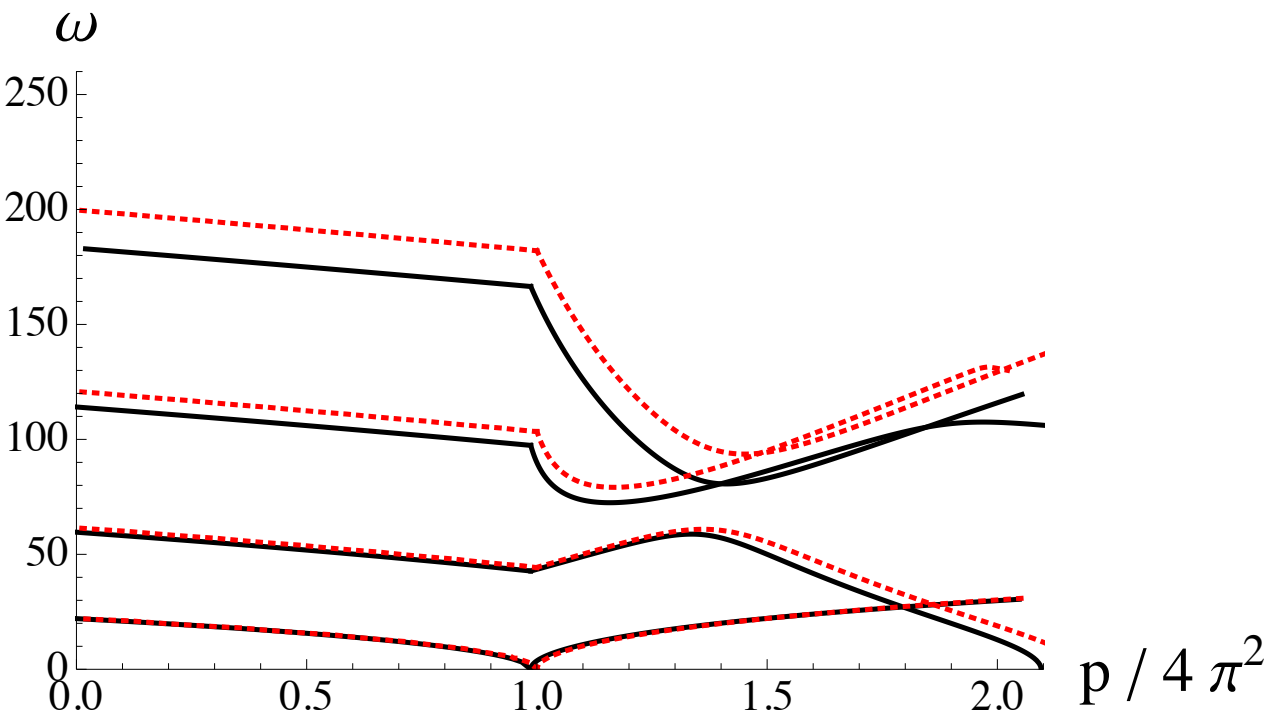

Figure D.14: Frequencies for the lowest four vibration modes of a clamped-clamped rod around its fundamental and post-buckled equilibrium configurations, in load-control boundary conditions. Plain curves are for the extensible case with $\eta=1 / 4800$, and dotted (red) curves are for the inextensible case $(\eta=0)$.

[11] J.-J. Marigo, N. Meunier, Hierarchy of one-dimensional models in nonlinear elasticity, Journal of Elasticity 83 (2006) 1-28

[12] S. Woinowsky-Krieger, The effect of an axial force on the vibration of hinged bars, Journal Applied Mechanics 17 (1950) 35-36.

[13] N. Yamaki, A. Mori, Non-linear vibrations of a clamped beam with initial deflection and initial axial displacement, part I: Theory, Journal of Sound and Vibration 71 (1980) 333 - 346.

[14] N. Yamaki, K. Otomo, A. Mori, Non-linear vibrations of a clamped beam with initial deflection and initial axial displacement, part II: Experiment, Journal of Sound and Vibration 71 (1980) 347 - 360.

[15] A. Nayfeh, S. Emam, Exact solution and stability of postbuckling configurations of beams, Nonlinear Dynamics 54 (2008) 395-408.

[16] L. Euler, Methodus inveniendi lineas curvas maximi minimivi propreitate gaudentes, Opera Omnia I 24 (1744) 231-297.

[17] V. G. A. Goss, The history of the planar elastica: Insights into mechanics and scientific method, Science \& Education 18 (2009) 1057-1082.

[18] M. Nizette, A. Goriely, Toward a classification of Euler-Kirchhoff filaments, Journal of Mathematical Physics 40 (1999) 2830-2866. 